are similar regulations in many parts of the USA, Canada, and South Africa.

No such legislation exists in this country; it has been stated that the number of deaths is comparatively small and that it is difficult to justify safety regulations that affect private property. Accidents that leave a child dead or severely handicapped, however, are a tragedy, particularly when they most probably could have been prevented by simple safety measures. Although these deaths are a relatively small proportion of the 50 or so children aged under 5 who are drowned each year, ${ }^{5}$ they are preventable; it is surely not unreasonable, therefore, to require the owners of swimming pools, who are usually relatively wealthy, to ensure that they are safely enclosed.

We thank Mr R Ferguson of the Home Office and Mrs J Burton of the medical statistics unit, Office of Population Censuses and Surveys, for their help. The analysis and conclusions described in this article, however, are ours alone.

${ }^{1}$ Pearn J, Nixon J, Wilkey I. Freshwater drowning and near drowning accidents involving children. A five year total population study. $M e d \mathcal{F}$ Aust 1976;2:942-6.

2 Peterson B. Morbidity of childhood near drowning. Pediatrics 1977;59: 364-70

3 Thorson J, Fredin H, Gerdman P, Wiklund R. Accidental drowning among children in Sweden 1958-1967. Acta Socio-Medica Scandinavica $1970 ; 2-3: 149-59$

4 Pearn J, Thompson J. Drowning and near drowning in the Australian Capital Territory. A five year total population study of immersion accidents. Med $\mathcal{F}$ Aust $1977 ; 1: 130-3$.

5 Office of Population Censuses and Surveys. Mortality statistics 1979. Accidents and violence. London: HMSO, 1981.

(Accepted 19 May 1982)

All Saints' Hospital, Chatham, Kent

W BARRY, MB, MRCP, paediatric registrar (now at Belgrave Hospital, London SW9)

T M LITTLE, MB, MRCP, consultant paediatrician

Llandough Hospital, near Penarth, South Glamorgan

J R SIBERT, MD, MRCP, consultant paediatrician

\section{Multiple hepatic abscesses complicating continuous ambulatory peritoneal dialysis}

Peritonitis is common during continuous ambulatory peritoneal dialysis $^{12}$ and may prove lethal. ${ }^{3}$ We report a case of relapsing peritonitis complicated by multiple hepatic abscesses.

\section{Case report}

A 41-year-old man had been receiving haemodialysis from 1970 to 1977 . Continuous ambulatory peritoneal dialysis was started in March 1980 for terminal renal failure due to chronic rejection causing nephrotic syndrome. During the first nine months of continuous ambulatory peritoneal dialysis three episodes of peritonitis were recorded (overall incidence at this unit = one episode every 16 patient-months). After 11 months the patient was admitted to hospital for peritonitis; culture grew no pathogens. Continuous peritoneal lavage with cefalexin, in association with parenteral cefalexin and tobramycin, resulted in clinical improvement and after three days continuous ambulatory peritoneal dialysis was started again with the same antibiotic treatment. Despite the persistence of slight fever, antibiotics were stopped on the 14th day. Three days later, epigastric pain returned, peritoneal effluent became cloudy, and culture was positive for Staphylococcus albus, sensitive to cephalosporins. Intraperitoneal and parenteral treatment were given in accordance with "antibiogram," but despite initial relief abdominal pain reappeared after 11 days, localised in the right upper quadrant, radiating to the shoulder; palpation elicited extreme pain at the hepatic border, localised at the intersection of the outer edge of the rectus abdominis. Peritoneal fluid was purulent with a high leucocyte count; intramuscular tobramycin was started. Abdominal echotomography gave negative results.

Nineteen days after operation the patient underwent exploratory laparotomy. A corpuscolate fluid was found in the peritoneal cavity and a small area $(8 \times 3 \mathrm{~cm})$ of swelling and reddening with microabscesses was found at the lower side of the right hepatic lobe, along the posterior edge (segments V, VII, VIII). Exploration puncture gave scant bloody fluid, but culture was positive for Staph albus. The abscesses were too small (1-4 $\mathrm{mm}$ in diameter) to warrant surgical drainage, so the Tenkhoff catheter was removed and replaced by a stylet catheter. Antibiotic treatment included intraperitoneal and intravenous vancomycin and intravenous amikacin for three weeks, and erythromycin orally for four weeks. From the second postoperative day the patient underwent haemodialysis through an external femoral bypass. Thirteen days after operation hepatic scintigraphy showed no alteration attributable to abscess and the patient was discharged without antibiotic treatment. Twelve months after discharge the patient is undergoing haemodialysis and is rehabilitated to grade A 1 (code of the European Dialysis and Transplant Association).

\section{Comment}

Gram-positive micro-organisms, particularly Staph albus, are the most frequent causal agent of peritonitis during continuous ambulatory peritoneal dialysis. ${ }^{2}$ Generally, these agents are responsive to cephalosporins, which are used as initial treatment until the results of bacteriological culture are available. ${ }^{2}$ The case described here illustrates that peritonitis due to Staph albus may lead to severe infectious complications, such as hepatic abscess. This patient presented predisposing factors such as nephrotic syndrome and a long period of immunosuppressive treatment before starting continuous ambulatory peritoneal dialysis. We believe that antibiotics were discontinued too soon, before the septic focus in the liver had been eradicated. Staphylococci are not usually the cause of hepatic abscesses. ${ }^{4}$ In the case described micro-organisms presumably reached the liver through the portal vein, though no such occurrence has been reported in patients undergoing continuous ambulatory peritoneal dialysis.

We recommend exploratory laparotomy for making a prompt diagnosis when other methods fail to show the cause of a persisting peritonitis. ${ }^{5}$ This may be of considerable help in establishing appropriate treatment for diseases such as hepatic abscesses which have a poor prognosis. Multiple hepatic abscesses do not lend themselves to adequate surgical drainage, and, though they can sometimes be evacuated by syringe, an aggressive schedule of specific antibiotic treatment maintained for a sufficiently long period remains the best possible way of achieving a cure.

1 Rubin J, Rogers WA, Taylor HM, et al. Peritonitis during continuous ambulatory dialysis. Ann Intern Med 1980;92:7-13.

2 Vas SI, Low DE, Oreopulos DG. Peritonitis. In: Nolph KD, ed. Peritoneal dialysis. The Hague: Martinus Nijhoff, 1981:344-65.

3 Oreopulos DG, Vas S, Khanna R. Treatment of peritonitis. In: Moncrief JW, Popowich RP, eds. CAPD update. New York: Masson Publishing, $1981: 259-64$

${ }^{4}$ Palmer ED. The changing manifestations of pyogenic liver abscess. fAMA $1975 ; 231: 192-4$.

5 Fridday RO, Barriga P, Crummy AB. Detection and localization of intraabdominal abscesses by diagnostic ultrasound. Arch Surg 1975;110: 335-8.

(Accepted 24 May 1982)

Renal Unit, San Carlo Borromeo Hospital, 20153 Milan, Italy

L LUCIANI, MD, assistant

M G GENTILE, MD, assistant

B SCARDUELLI, $M D$, assistant

$R$ A SINICO, $\mathrm{MD}$, assistant

G D'AMICO, MD, head

Department of Surgery, San Carlo Borromeo Hospital, 20153 Milan, Italy

G SAMORI, MD, assistant

\section{Myxoedema coma induced by beta-adrenoreceptor-blocking agent}

Although the pathogenesis of myxoedema coma is uncertain, precipitating factors such as exposure to cold, infection, administration of central nervous system depressants, alveolar hypoventilation, and hyponatraemia are reported to predispose to its development. Myxoedema coma induced by administration of beta-adrenoreceptorblocking agents has not, however, been reported. We describe a patient with myxoedema coma induced by a beta-blocker who recovered consciousness after withdrawal of the drug. 